Revue d'histoire de l'Amérique française

RAS REVUE D.HISTOIRE DE L'AMÉRIQUE FRANÇAISE

\title{
Un catalogue général des manuscrits de Lionel Groulx
}

\section{Robert Desaulniers}

Volume 36, numéro 1, juin 1982

URI : https://id.erudit.org/iderudit/304044ar

DOI : https://doi.org/10.7202/304044ar

Aller au sommaire du numéro

Éditeur(s)

Institut d'histoire de l'Amérique française

ISSN

0035-2357 (imprimé)

1492-1383 (numérique)

Découvrir la revue

Citer ce document

Desaulniers, R. (1982). Un catalogue général des manuscrits de Lionel Groulx. Revue d'histoire de l'Amérique française, 36(1), 155-157.

https://doi.org/10.7202/304044ar d'utilisation que vous pouvez consulter en ligne.

https://apropos.erudit.org/fr/usagers/politique-dutilisation/ 


\section{UN CATALOGUE GÉNÉRAL DES MANUSCRITS DE LIONEL GROULX}

L'oeuvre imprimée et inédite de Lionel Groulx étonne par sa dimension et sa variété. Une bio-bibliographie, publiée en 1964 et intitulée L'oeuvre du chanoine Lionel Groulx ${ }^{1}$, réussit à peine en 197 pages à en énumérer tous les titres. Elle révèle néanmoins une quarantaine d'ouvrages historiques et de fiction, plus de deux cents articles de revues et de journaux, autant de comptes rendus, au moins soixante-douze discours et allocutions et près de deux cents conférences. Elle fait état également d'une quarantaine de cours donnés par Groulx et d'un grand nombre d'écrits divers, poèmes, préfaces et ouvrages en collaboration.

Au cours de ses quelque soixante-dix ans de production littéraire et historique, l'homme s'est très peu défait de ses manuscrits. Ses brouillons, versions, cahiers de notes, écrits personnels et sa correspondance sont demeurés, au même titre que ses livres, des compagnons précieux, fréquemment visités.

Quinze ans après la disparition de l'écrivain, aucun instrument de recherche ne fournit encore une image exacte des dimensions de ce fonds unique, ni n'en montre tout l'intérêt pour la recherche.

Bien que réunis pour la plupart sous un même toit - celui de la Fondation Lionel-Groulx - et conservés dans les meilleures conditions, les manuscrits de Groulx, tels qu'ils se présentent, exigent des chercheurs une consultation laborieuse dont les résultats risquent d'être décevants. Au-delà de la quantité des pièces et de l'absence d'un inventaire, la nature même des documents et les exigences ou les circonstances de leur rédaction et de leur conservation nuisent à la vision globale du fonds et en masquent l'intérêt pour les divers champs de recherche.

En effet, brouillons, notes de recherche, versions définitives et relations diverses ont été rédigés, au hasard des besoins et des moyens du moment, sur des feuillets de tout format et de tout

\footnotetext{
Juliette Rémillard et Madeleine Dionne, L'oeuvre du chanoine Lionel Groulx (avant-propos de Victor Barbeau) (Académie canadienne-française, Montréal, 1957), $197 \mathrm{p}$.
} 
genre (à l'occasion des bouts de papier), dans des cahiers et des calepins souvent composites et dans lesquels l'ordre des textes n'est pas toujours chronologique. Certains manuscrits ont été insérés dans des spicilèges (au nombre de 150), d'autres, encartés dans des livres, d'autres enfin, réunis en liasses ou à l'intérieur de cartons. À ces particularités s'ajoutent les difficultés usuelles de l'identification: absence de titre, titres provisoires, datation irrégulière, imprécise, absence ou erreurs de pagination.

Dans de telles circonstances, propres sans doute aux fonds privés et familières aux archives d'hommes de lettres, les liens entre certains documents, même après classification, ne sont pas toujours évidents et de ce fait, la filiation des idées, l'évolution de la pensée, la contribution des lectures et des rencontres, les remaniements de texte et les repentirs d'auteur sont plus difficilement saisissables et risquent d'échapper aux plus vigilantes investigations.

Afin de mettre cette masse archivistique au service de la recherche savante, un projet de catalogue général des manuscrits de Lionel Groulx s'est mis en marche en octobre 1981, grâce à l'initiative d'une équipe de chercheurs de l'Institut d'études médiévales de l'Université de Montréal et au soutien financier du Conseil de recherches en sciences humaines du Canada.

Encore peu répandu en Amérique du Nord, le catalogue de manuscrits est un instrument de recherche d'une grande précision qui répond davantage à un besoin scientifique qu'à un besoin pratique d'identification et d'organisation de la matière. Originellement élaboré pour la description des manuscrits du Moyen âge, le catalogue s'adapte avec souplesse aux divers besoins de la recherche érudite et sert particulièrement bien les gros corpus. Ses objectifs sont d'offrir pour chaque article du corpus examiné des informations complètes et dans la mesure du possible définitives et, grâce à son système de notes, de renvois et d'index, de fournir aux chercheurs des pistes de recherche.

Dans cette optique, le Catalogue général des manuscrits de Lionel Groulx (CMG) fournira une description systématique des manuscrits dans tous les fonds connus. Chaque notice catalographique sera accompagnée d'une cote qui permettra de localiser rapidement le document décrit.

Nous aurons l'occasion de préciser, dans d'autres chroniques, la méthodologie du catalogue de manuscrits. Contentons-nous de signaler ici qu'il est idéalement exhaustif dans l'aspect du listage. Même après publication, il demeure ouvert et des suppléments sont prévus pour rendre compte des découvertes sans que la valeur de l'ouvrage principal n'en soit diminuée. 
Essentiellement descriptif, son intérêt se porte d'abord sur l'identification du manuscrit. Il présente de façon uniforme les données relatives au titre, à la responsabilité, à la date, à la collation et à l'état de conservation. Au besoin, le catalographe s'inspire de la démarche de la codicologie savante pour retracer l'histoire même du support.

À la description matérielle s'ajoutent des informations sur la nature et la portée du manuscrit. Selon le cas, le catalographe notera certaines particularités du message écrit, en établira les liens avec d'autres pièces du corpus, en signalera la place dans l'ensemble de l'oeuvre, de la production ou dans la vie de l'auteur.

Dans la plupart des cas, la notice se complète par le résumé et le dépouillement du contenu.

L'intérêt d'un tel instrument d'investigation débordera facilement le personnage et l'oeuvre sur lesquels il se penche. Le seul aspect de la méthodologie qui nécessitera inévitablement une adaptation au contexte d'une production manuscrite nord-américaine et récente suscitera sans doute une réflexion sur la pertinence des instruments de recherche usuels face aux besoins toujours plus exigeants d'une recherche axée de plus en plus sur l'interdisciplinarité.

D'autre part, l'édition critique des inédits de Groulx, déjà commencée, saura certainement mettre à profit les ressources d'un inventaire aussi précis.

Le CMG aura à tout le moins le mérite de rendre disponible une documentation demeurée forcément jusque-là le privilège de quelques spécialistes, d'éliminer les pertes de temps des annotations et des dépouillements individuels et de mettre à jour des manuscrits qui, à cause de leur anonymat, de leur format ou de leur regroupement accidentel, seraient demeurés à jamais ignorés des chercheurs. 